\title{
New Characterizations of Musielak-Orlicz-Sobolev Spaces via Sharp Ball Averaging Functions
}

\author{
Sibei Yang, Dachun Yang* and Wen Yuan
}

\begin{abstract}
In this article, the authors establish a new characterization of the Musielak-OrliczSobolev space on $\mathbb{R}^{n}$, which includes the classical Orlicz-Sobolev space, the weighted Sobolev space and the variable exponent Sobolev space as special cases, in terms of sharp ball averaging functions. Even in a special case, namely, the variable exponent Sobolev space, the obtained result in this article improves the corresponding result obtained by P. Hästö and A. M. Ribeiro [Commun. Contemp. Math. 19 (2017), 1650022, 13 pp] via weakening the assumption $f \in L^{1}\left(\mathbb{R}^{n}\right)$ into $f \in L_{\text {loc }}^{1}\left(\mathbb{R}^{n}\right)$, which was conjectured to be true by Hästö and Ribeiro in the aforementioned same article.
\end{abstract}

\section{Introduction}

Let $s \in(0,1)$ and $p \in[1, \infty)$. In what follows, we use the symbol $L^{p}\left(\mathbb{R}^{n}\right)$ to denote the space of all Lebesgue measurable functions $f$ on $\mathbb{R}^{n}$ such that

$$
\|f\|_{L^{p}\left(\mathbb{R}^{n}\right)}:=\left[\int_{\mathbb{R}^{n}}|f(x)|^{p} d x\right]^{1 / p}<\infty
$$

and $L^{p}\left(\mathbb{R}^{n} \times \mathbb{R}^{n}\right)$ is similarly defined via replacing $\mathbb{R}^{n}$ by $\mathbb{R}^{n} \times \mathbb{R}^{n}$. The fractional order Sobolev space $W^{s, p}\left(\mathbb{R}^{n}\right)$ is defined by setting

$$
W^{s, p}\left(\mathbb{R}^{n}\right):=\left\{f \in L^{p}\left(\mathbb{R}^{n}\right): \frac{f(x)-f(y)}{|x-y|^{\frac{n}{p}+s}} \in L^{p}\left(\mathbb{R}^{n} \times \mathbb{R}^{n}\right)\right\}
$$

equipped with the norm

$$
\|f\|_{W^{s, p}\left(\mathbb{R}^{n}\right)}:=\left\{\int_{\mathbb{R}^{n}} \int_{\mathbb{R}^{n}} \frac{|f(x)-f(y)|^{p}}{|x-y|^{n+s p}} d x d y\right\}^{1 / p} .
$$

Bourgain, Brezis and Mironescu [6, 7] studied the limit behavior of the norm $\|\cdot\|_{W^{s, p}\left(\mathbb{R}^{n}\right)}$ as $s \uparrow 1$, here and hereafter, the symbol $s \uparrow 1$ means that $s \in(0,1)$ increasingly converges to 1 . More precisely, Bourgain, Brezis and Mironescu [6] showed that, for any $f \in L^{p}\left(\mathbb{R}^{n}\right)$, it holds true that

$$
\lim _{s \uparrow 1}(1-s) \int_{\mathbb{R}^{n}} \int_{\mathbb{R}^{n}} \frac{|f(x)-f(y)|^{p}}{|x-y|^{n+s p}} d x d y=C_{(n, p)} \int_{\mathbb{R}^{n}}|\nabla f(x)|^{p} d x
$$

2010 Mathematics Subject Classification. Primary 46E35; Secondary 42B35, 42B25.

Key words and phrases. Musielak-Orlicz-Sobolev space, variable exponent Sobolev space, Orlicz-Sobolev space, sharp ball averaging function.

This work is supported by the National Natural Science Foundation of China (Grant Nos. 11871254, 11571289, $11571039,11761131002,11726621,11671185$ and 11871100).

${ }^{*}$ Corresponding author/October 4, 2018/Final version. 
where $C_{(n, p)}$ is an explicit positive constant depending on $n$ and $p$. Recently, these results have been generalized to the cases of the so-called magnetic space [36] and the Orlicz-Sobolev space [17].

In what follows, we use the symbol $L_{\text {loc }}^{1}\left(\mathbb{R}^{n}\right)$ to denote the space of all locally integrable functions on $\mathbb{R}^{n}$. Let $\mathcal{P}\left(\mathbb{R}^{n}\right)$ be the set of all measurable functions $p: \mathbb{R}^{n} \rightarrow[1, \infty)$. For any $p \in \mathcal{P}\left(\mathbb{R}^{n}\right)$, let

$$
p^{+}:=\underset{x \in \mathbb{R}^{n}}{\operatorname{ess} \sup } p(x) \text { and } p^{-}:=\underset{x \in \mathbb{R}^{n}}{\operatorname{ess} \inf } p(x) .
$$

For any given $p \in \mathcal{P}\left(\mathbb{R}^{n}\right)$, the variable exponent modular $\rho_{p(\cdot)}$ is defined by setting, for any $f \in$ $L_{\text {loc }}^{1}\left(\mathbb{R}^{n}\right)$,

$$
\rho_{p(\cdot)}(f):=\int_{\mathbb{R}^{n}}|f(x)|^{p(x)} d x .
$$

The variable exponent Lebesgue space $L^{p(\cdot)}\left(\mathbb{R}^{n}\right)$ is defined by setting

$$
\begin{aligned}
& L^{p(\cdot)}\left(\mathbb{R}^{n}\right):=\left\{f \text { is measurable on } \mathbb{R}^{n}:\right. \\
&\text { there exists } \left.\lambda \in(0, \infty) \text { such that } \rho_{p(\cdot)}(\lambda f)<\infty\right\}
\end{aligned}
$$

equipped with the Luxemburg (also called the Luxembourg-Nakano) norm

$$
\|f\|_{L^{p(\cdot)}\left(\mathbb{R}^{n}\right)}:=\inf \left\{\lambda \in(0, \infty): \rho_{p(\cdot)}\left(\frac{f}{\lambda}\right) \leq 1\right\} .
$$

Moreover, the variable exponent Sobolev space $W^{1, p(\cdot)}\left(\mathbb{R}^{n}\right)$ is defined by setting

$$
W^{1, p(\cdot)}\left(\mathbb{R}^{n}\right):=\left\{u \in L^{p(\cdot)}\left(\mathbb{R}^{n}\right):|\nabla u| \in L^{p(\cdot)}\left(\mathbb{R}^{n}\right)\right\} .
$$

For more studies on variable exponent Lebesgue spaces and Sobolev spaces, we refer the reader to $[11,14]$.

However, in the case of variable exponent Sobolev spaces, the similar characterization as in $[6,17,36]$ does not hold true, because that the translation operator may not be bounded on the variable exponent Lebesgue space. Indeed, from [14, Proposition 3.6.1], it follows that the translation operator is bounded on the variable exponent Lebesgue space $L^{p(\cdot)}\left(\mathbb{R}^{n}\right)$ if and only if $p$ is a constant. In particular, this means that, in the different quotient appearing in (1.1), we can not replace the constant exponent $p$ simply by the variable exponent $p(x)$ or $p(y)$. The same problem also appears in [19]. Instead of this, Diening and Hästö [16] creatively replaced the different quotient in the definition of the trace space by the sharp averaging operator $M_{B}^{\sharp}$ (see, for example, $[25,(1.3)]$ or (1.4) below). Motivated by the work [16], under the assumptions that the variable exponent $p(\cdot)$ satisfies the local log-Hölder continuity condition, the log-Hölder decay condition (at infinity) and $p^{-} \in(1, \infty)$ with $p^{-}$as in (1.2), Hästö and Ribeiro [25, Theorem 4.1] obtained a new characterization of the variable exponent Sobolev space in terms of the sharp averaging operator, which is re-stated as Theorem 1.A below.

As a natural generalization of the Lebesgue space, the Orlicz space was introduced by Birnbaum and Orlicz [5] and Orlicz [33]. Since then, the theory of Orlicz spaces has been well developed and these spaces have been widely used in probability, statistics, potential theory, partial 
differential equations, as well as harmonic analysis and some other fields of analysis (see, for example, $[2,9,30,29,35,34])$. Later, Musielak [28] introduced the so-called Musielak-Orlicz space, which contains the Orlicz space and the weighted Lebesgue space as special cases. Nowadays, the theory of both Musielak-Orlicz spaces and function spaces of Musielak-Orlicz type has been well developed and theses spaces have been widely used in many branches of mathematics. It is worth pointing out that Musielak-Orlicz spaces or Musielak-Orlicz-Sobolev spaces naturally appear in the study of the regularity for solutions of some nonlinear elliptic equations or minimizers of functionals with non-standard growth (see, for example, $[1,2,9,10]$ ). We also refer the reader to [31, 32, 38, 39] for some recent progresses about the real-variable theory of both Musielak-Orlicz-Sobolev spaces and function spaces of Musielak-Orlicz type.

In this article, motivated by $[16,17,25]$, we obtain a new characterization of the MusielakOrlicz-Sobolev space on $\mathbb{R}^{n}$, including the classical Orlicz-Sobolev space, the weighted Sobolev space and the variable exponent Sobolev space, in terms of sharp ball averaging functions. Even in the special case, namely, the variable exponent Sobolev space, the obtained result in this article improves the corresponding result obtained in [25, Theorem 4.1] via weakening the assumption $f \in L^{1}\left(\mathbb{R}^{n}\right)$ into $f \in L_{\text {loc }}^{1}\left(\mathbb{R}^{n}\right)$, which positively confirms a conjecture proposed by Hästö and Ribeiro in [25, Remark 4.1].

To describe the main result of this article, we first recall some necessary notions and notation.

Definition 1.1. (i) A function $G:[0, \infty) \rightarrow[0, \infty)$ is called an Orlicz function if it satisfies the following conditions:

(i) ${ }_{1} G$ is continuous, convex, increasing and $G(0)=0$;

(i) $\lim _{t \rightarrow 0^{+}} \frac{G(t)}{t}=0$ and $\lim _{t \rightarrow \infty} \frac{G(t)}{t}=\infty$.

(ii) A function $\Phi: \mathbb{R}^{n} \times[0, \infty) \rightarrow[0, \infty)$ is called a Musielak-Orlicz function if, for any given growth variable $t \in[0, \infty), \Phi(\cdot, t)$ is measurable and, for almost every space variable $x \in \mathbb{R}^{n}$, the function $\Phi(x, \cdot)$ is an Orlicz function.

(iii) Let $\Phi$ be a Musielak-Orlicz function. Then the complementary function of $\Phi$, denoted by $\Phi^{*}$, is defined by setting, for any $x \in \mathbb{R}^{n}$ and $s \in[0, \infty)$,

$$
\Phi^{*}(x, s):=\sup _{t \in[0, \infty)}\{s t-\Phi(x, t)\} .
$$

The Musielak-Orlicz space $L^{\Phi}\left(\mathbb{R}^{n}\right)$ and the Musielak-Orlicz-Sobolev space $W^{1, \Phi}\left(\mathbb{R}^{n}\right)$ are defined as follows.

Definition 1.2. Let $\Phi$ be a Musielak-Orlicz function. For any given $f \in L_{\mathrm{loc}}^{1}\left(\mathbb{R}^{n}\right)$, the MusielakOrlicz modular of $f$ is defined by setting

$$
\rho_{\Phi}(f):=\int_{\mathbb{R}^{n}} \Phi(x,|f(x)|) d x .
$$

Then the Musielak-Orlicz space $L^{\Phi}\left(\mathbb{R}^{n}\right)$ is defined by setting

$$
L^{\Phi}\left(\mathbb{R}^{n}\right):=\left\{u \text { is measurable on } \mathbb{R}^{n}:\right.
$$


there exists $\lambda \in(0, \infty)$ such that $\left.\rho_{\Phi}(\lambda f)<\infty\right\}$

equipped with the Luxemburg (also called the Luxembourg-Nakano) norm

$$
\|u\|_{L^{\Phi}\left(\mathbb{R}^{n}\right)}:=\inf \left\{\lambda \in(0, \infty): \int_{\mathbb{R}^{n}} \Phi\left(x, \frac{|u(x)|}{\lambda}\right) d x \leq 1\right\} .
$$

Moreover, the Musielak-Orlicz-Sobolev space $W^{1, \Phi}\left(\mathbb{R}^{n}\right)$ is defined by setting

$$
W^{1, \Phi}\left(\mathbb{R}^{n}\right):=\left\{u \in L^{\Phi}\left(\mathbb{R}^{n}\right):|\nabla u| \in L^{\Phi}\left(\mathbb{R}^{n}\right)\right\}
$$

equipped with the norm

$$
\|u\|_{W^{1, \Phi}\left(\mathbb{R}^{n}\right)}:=\|u\|_{L^{\Phi}\left(\mathbb{R}^{n}\right)}+\|\| \nabla u \|_{L^{\Phi}\left(\mathbb{R}^{n}\right)},
$$

where $\|\mid \nabla u\|_{L^{\Phi}\left(\mathbb{R}^{n}\right)}$ is defined via replacing $u$ by $|\nabla u|$ in (1.3).

Furthermore, the homogeneous Musielak-Orlicz Sobolev space $\dot{W}^{1, \Phi}\left(\mathbb{R}^{n}\right)$ is defined by setting

$$
\dot{W}^{1, \Phi}\left(\mathbb{R}^{n}\right):=\left\{u \in L_{\mathrm{loc}}^{1}\left(\mathbb{R}^{n}\right):|\nabla u| \in L^{\Phi}\left(\mathbb{R}^{n}\right)\right\}
$$

equipped with the norm $\|u\|_{\dot{W}^{1, \Phi}\left(\mathbb{R}^{n}\right)}:=\|\| \nabla u \|_{L^{\Phi}\left(\mathbb{R}^{n}\right)}$.

In what follows, for any $x \in \mathbb{R}^{n}$ and $r \in(0, \infty)$, we always let

$$
B(x, r):=\left\{y \in \mathbb{R}^{n}:|y-x|<r\right\}
$$

be a ball of $\mathbb{R}^{n}$ with the center $x$ and the radius $r$.

Definition 1.3. Let $\left\{\psi_{\epsilon}\right\}_{\epsilon>0}$ be a family of functions in $L^{1}([0,1])$ such that, for any $\epsilon \in(0, \infty)$,

$$
\psi_{\epsilon} \geq 0, \quad \int_{0}^{1} \psi_{\epsilon}(r) d r=1
$$

and, for any $\gamma \in(0,1)$,

$$
\lim _{\epsilon \rightarrow 0^{+}} \int_{\gamma}^{1} \psi_{\epsilon}(r) d r=0
$$

here and hereafter, the symbol $\epsilon \rightarrow 0^{+}$means that $\epsilon \in(0, \infty)$ and $\epsilon \rightarrow 0$.

Assume that $\Phi$ is a Musielak-Orlicz function. For any $\epsilon \in(0, \infty)$ and $f \in L_{\text {loc }}^{1}\left(\mathbb{R}^{n}\right)$, let

$$
\rho_{\sharp}^{\epsilon}(f):=\int_{0}^{1}\left[\int_{\mathbb{R}^{n}} \Phi\left(x, \frac{1}{r} M_{B(x, r)}^{\sharp}(f)\right) d x\right] \psi_{\epsilon}(r) d r,
$$

where the sharp ball averaging function $M_{B(x, r)}^{\sharp}(f)$ of $f$ is defined by setting, for any $f \in L_{\text {loc }}^{1}\left(\mathbb{R}^{n}\right)$, $x \in \mathbb{R}^{n}$ and $r \in(0, \infty)$,

$$
M_{B(x, r)}^{\sharp}(f):=\frac{1}{|B(x, r)|} \int_{B(x, r)}\left|f(y)-f_{B(x, r)}\right| d y
$$

and

$$
f_{B(x, r)}:=\frac{1}{|B(x, r)|} \int_{B(x, r)} f(y) d y
$$


Recall that $M_{B(x, r)}^{\sharp}$ is also called the sharp averaging operator in [25, p. 1650022-2]. Moreover, for any given $\epsilon \in(0, \infty)$ and $f \in L_{\text {loc }}^{1}\left(\mathbb{R}^{n}\right)$, the norm $\|f\|_{\Phi, \sharp}^{\epsilon}$ is defined by setting

$$
\|f\|_{\Phi, \sharp}^{\epsilon}:=\inf \left\{\lambda \in(0, \infty): \rho_{\sharp}^{\epsilon}\left(\frac{f}{\lambda}\right) \leq 1\right\} .
$$

We point out that several examples of such families of functions $\left\{\psi_{\epsilon}\right\}_{\epsilon>0}$ as in Definition 1.3 were given by Brezis [8, Remark 8].

Recall that a function $p: \mathbb{R}^{n} \rightarrow \mathbb{R}$ is said to satisfy the local log-Hölder continuity condition if there exists a positive constant $C$ such that, for any $x, y \in \mathbb{R}^{n}$ with $x \neq y$,

$$
|p(x)-p(y)| \leq \frac{C}{\log \left(e+\frac{1}{|x-y|}\right)} ;
$$

a function $p: \mathbb{R}^{n} \rightarrow \mathbb{R}$ is said to satisfy the log-Hölder decay condition (at infinity) if there exist positive constants $C \in(0, \infty)$ and $p_{\infty} \in[1, \infty)$ such that, for any $x \in \mathbb{R}^{n}$,

$$
\left|p(x)-p_{\infty}\right| \leq \frac{C}{\log (e+|x|)} .
$$

If a function $p$ satisfies both the local log-Hölder continuity condition and the log-Hölder decay condition, then the function $p$ is said to satisfy the log-Hölder continuity condition. It is easy to see that, if $p$ satisfies the log-Hölder continuity condition, then $p$ is bounded and $p^{+}<\infty$ with $p^{+}$ as in (1.2).

For the variable exponent Sobolev space, the following conclusion was established in [25, Theorem 4.1].

Theorem 1.A. Let $p \in \mathcal{P}\left(\mathbb{R}^{n}\right)$ satisfy the log-Hölder continuity condition and $p^{-} \in(1, \infty)$ with $p^{-}$ as in (1.2), and $\left\{\psi_{\epsilon}\right\}_{\epsilon>0}$ be a family of functions as in Definition 1.3. For any $x \in \mathbb{R}^{n}$ and $t \in[0, \infty)$, let $\Phi(x, t):=t^{p(x)}$. Assume further that $f \in L^{1}\left(\mathbb{R}^{n}\right)$. Then $|\nabla f| \in L^{p(\cdot)}\left(\mathbb{R}^{n}\right)$ if and only if

$$
\limsup _{\epsilon \rightarrow 0^{+}} \rho_{\sharp}^{\epsilon}(f)<\infty,
$$

here and hereafter, $\nabla f$ denotes the gradient of $f$. In this case,

$$
\lim _{\epsilon \rightarrow 0^{+}} \rho_{\sharp}^{\epsilon}(f)=\rho_{p(\cdot)}\left(c_{0}|\nabla f|\right)
$$

and

$$
\lim _{\epsilon \rightarrow 0^{+}}\|f\|_{\Phi, \sharp}^{\epsilon}=c_{0}\|\| \nabla f \|_{L^{p(\cdot)}\left(\mathbb{R}^{n}\right)},
$$

where $c_{0}:=\frac{1}{\left|B\left(\overrightarrow{0}_{n}, 1\right)\right|} \int_{B\left(\overrightarrow{0}_{n}, 1\right)}\left|x \cdot e_{1}\right| d x$ with $\overrightarrow{0}_{n}$ being the origin of $\mathbb{R}^{n}$ and $e_{1}:=(1, \overbrace{0, \ldots, 0}^{n-1 \text { times }})$.

Remark 1.4. We point out that, in [25, Remark 4.1], Hästö and Ribeiro also conjectured that Theorem 1.A still holds true if $f \in L^{1}\left(\mathbb{R}^{n}\right)$ is replaced by $f \in L_{\text {loc }}^{1}\left(\mathbb{R}^{n}\right)$, which is confirmed by Corollary 1.12(i) below, as a simple corollary of Theorem 1.10 below. 
To state the main result of this article, we first give some assumptions on the Musielak-Orlicz function $\Phi$.

Assumption 1.5. The Musielak-Orlicz function $\Phi$ is locally integrable on the space variable in $\mathbb{R}^{n}$, namely, for any given positive constant $c$ and any compact set $K \subset \mathbb{R}^{n}$, we have

$$
\int_{K} \Phi(x, c) d x<\infty
$$

Assumption 1.6. The Musielak-Orlicz function $\Phi$ satisfies the $\Delta_{2}$-condition on the growth variable, namely, there exists a positive constant $\kappa \in(1, \infty)$ such that, for almost every $x \in \mathbb{R}^{n}$ and any $s \in[0, \infty)$,

$$
\Phi(x, 2 s) \leq \kappa \Phi(x, s) .
$$

Assumption 1.7. The Musielak-Orlicz function $\Phi$ has the property: $C_{c}^{\infty}\left(\mathbb{R}^{n}\right)$ is dense in the homogeneous Musielak-Orlicz-Sobolev space $\dot{W}^{1, \Phi}\left(\mathbb{R}^{n}\right)$ with respect to the norm $\|\cdot\|_{\dot{W}^{1, \Phi}\left(\mathbb{R}^{n}\right)}$, where the symbol $C_{c}^{\infty}\left(\mathbb{R}^{n}\right)$ denotes the set of all $C^{\infty}$ functions on $\mathbb{R}^{n}$ with compact supports.

Assumption 1.8. The Hardy-Littlewood maximal operator $M$ is bounded on the Musielak-Orlicz space $L^{\Phi}\left(\mathbb{R}^{n}\right)$, namely, there exists a positive constant $C$ such that, for any $f \in L^{\Phi}\left(\mathbb{R}^{n}\right)$,

$$
\|M(f)\|_{L^{\Phi}\left(\mathbb{R}^{n}\right)} \leq C\|f\|_{L^{\Phi}\left(\mathbb{R}^{n}\right)} .
$$

Here and hereafter, $M(f)$ denotes the Hardy-Littlewood maximal function of $f$, which is defined by setting, for any given $f \in L_{\text {loc }}^{1}\left(\mathbb{R}^{n}\right)$ and $x \in \mathbb{R}^{n}$,

$$
M(f)(x):=\sup _{x \in B} \frac{1}{|B|} \int_{B}|f(y)| d y,
$$

where the supremum is taken over all balls $B \subset \mathbb{R}^{n}$ containing $x$.

Remark 1.9. Here we give some examples of Musielak-Orlicz functions satisfying Assumptions 1.7 and 1.8 as follows.

(i) For any $x \in \mathbb{R}^{n}$ and $t \in[0, \infty)$, let $\Phi(x, t):=t^{p(x)}$, where $p(\cdot)$ is as in Theorem 1.A. It is known that $\Phi$ satisfies Assumptions 1.7 and 1.8 (see, for example, [14, Theorems 9.1.6 and 4.3.8]).

(ii) For any $x \in \mathbb{R}^{n}$ and $t \in[0, \infty)$, let $\Phi(x, t):=\varphi(t)$, where $\varphi$ is an Orlicz function satisfying the $\Delta_{2}$-condition. It is well known that Assumption 1.7 holds true for such $\Phi$ (see, for example, [3, Theorem 8.31]). Moreover, by [20, Theorem 2.1] (see also [26, Theorem 1.2.1]), we find that $\Phi$ satisfies Assumption 1.8.

(iii) Let $p \in[1, \infty)$. Recall that an almost everywhere non-negative and locally integrable function $\omega$ on $\mathbb{R}^{n}$ is called an $A_{p}\left(\mathbb{R}^{n}\right)$ weight if

$$
[\omega]_{A_{p}\left(\mathbb{R}^{n}\right)}:=\sup _{B \subset \mathbb{R}^{n}}\left\{\frac{1}{|B|} \int_{B} w(x) d x\right\}\left\{\frac{1}{|B|} \int_{B}[w(x)]^{-\frac{1}{p-1}} d x\right\}^{p-1}<\infty
$$


when $p \in(1, \infty)$, and

$$
[\omega]_{A_{1}\left(\mathbb{R}^{n}\right)}:=\sup _{B \subset \mathbb{R}^{n}}\left\{\frac{1}{|B|} \int_{B} w(x) d x\right\}\{\underset{y \in B}{\operatorname{essinf}} w(y)\}^{-1}<\infty,
$$

where the suprema are taken over all balls $B \subset \mathbb{R}^{n}$.

For any $x \in \mathbb{R}^{n}$ and $t \in[0, \infty)$, let $\Phi(x, t):=\omega(x) t^{q}$, where $q \in(1, \infty)$ and $\omega \in A_{q}\left(\mathbb{R}^{n}\right)$. From [37, Theorem 2.1.4], it follows that such $\Phi$ satisfies Assumption 1.7. Furthermore, it is well known that Assumption 1.8 holds true for $\Phi$ (see, for example, [21, Theorem 7.1.9]).

(iv) More examples of Musielak-Orlicz functions satisfying Assumption 1.7 were given in [4] (see also [39, Example 2.1]). Moreover, some necessary and sufficient conditions for the Musielak-Orlicz function $\Phi$ satisfying Assumption 1.8 were established in $[13,24]$.

Now we state the main result of this article as follows.

Theorem 1.10. Let $\Phi$ be a Musielak-Orlicz function satisfying Assumptions 1.5 through 1.8. Assume that the complementary function $\Phi^{*}$ to $\Phi$ satisfies Assumptions 1.5 and $1.6,\left\{\psi_{\epsilon}\right\}_{\epsilon>0}$ is a family of functions as in Definition 1.3 and $f \in L_{\mathrm{loc}}^{1}\left(\mathbb{R}^{n}\right)$. Then $|\nabla f| \in L^{\Phi}\left(\mathbb{R}^{n}\right)$ if and only if

$$
\limsup _{\epsilon \rightarrow 0^{+}} \rho_{\sharp}^{\epsilon}(f)<\infty .
$$

In this case,

$$
\lim _{\epsilon \rightarrow 0^{+}} \rho_{\sharp}^{\epsilon}(f)=\rho_{\Phi}\left(c_{0}|\nabla f|\right)
$$

and

$$
\lim _{\epsilon \rightarrow 0^{+}}\|f\|_{\Phi, \sharp}^{\epsilon}=c_{0}\|\| \nabla f \|_{L^{\Phi}\left(\mathbb{R}^{n}\right)},
$$

where $c_{0}$ is the same as in Theorem 1.A.

The detailed proof of Theorem 1.10 is presented in Section 2.

To show Theorem 1.10, we borrow some ideas from the proof of [25, Theorem 4.1]. More precisely, we first prove that (1.6) holds true for functions in $C_{c}^{\infty}\left(\mathbb{R}^{n}\right)$. Then, for a function $f \in$ $L_{\text {loc }}^{1}\left(\mathbb{R}^{n}\right)$ such that $|\nabla f| \in L^{\Phi}\left(\mathbb{R}^{n}\right)$, by the technique of approximation using functions from $C_{c}^{\infty}\left(\mathbb{R}^{n}\right)$ and some finer properties of Musielak-Orlicz functions, we show that (1.6) also holds true for such functions $f$. Moreover, from (1.6) and the properties of Musielak-Orlicz functions, we further deduce that (1.7) holds true. Comparing with [25, Theorem 4.1], instead of $f \in L^{1}\left(\mathbb{R}^{n}\right)$, we now only need to assume that $f \in L_{\mathrm{loc}}^{1}\left(\mathbb{R}^{n}\right)$ in Theorem 1.10 . To overcome the difficulty causing by this weaker assumption, in the proof of Theorem 1.10, we flexibly use the boundedness of the Hardy-Littlewood maximal operator on the Musielak-Orlicz space $L^{\Phi}\left(\mathbb{R}^{n}\right)$, which is assumed to hold true, and the subtle growth properties of Musielak-Orlicz functions obtained in Lemma 2.2 below. Moreover, we point out that the assumed boundedness of the Hardy-Littlewood maximal operator $M$ on the Musielak-Orlicz space $L^{\Phi}\left(\mathbb{R}^{n}\right)$ is known to hold true for several well-known Musielak-Orlicz functions $\Phi$; see Corollary 1.12 below.

As a simple conclusion of Theorem 1.10, we have the following conclusion, the details being omitted here. 
Corollary 1.11. Let $\Phi$ be a Musielak-Orlicz function satisfying Assumptions 1.5 through 1.8. Assume that the complementary function $\Phi^{*}$ to $\Phi$ satisfies Assumptions 1.5 and 1.6, $\left\{\psi_{\epsilon}\right\}_{\epsilon>0}$ is a family of functions as in Definition 1.3 and $f \in L_{\mathrm{loc}}^{1}\left(\mathbb{R}^{n}\right)$. Then $f \in W^{1, \Phi}\left(\mathbb{R}^{n}\right)$ if and only if $f \in L^{\Phi}\left(\mathbb{R}^{n}\right)$ and

$$
\limsup _{\epsilon \rightarrow 0^{+}} \rho_{\sharp}^{\epsilon}(f)<\infty .
$$

Moreover, if $f \in W^{1, \Phi}\left(\mathbb{R}^{n}\right)$, then

$$
\|f\|_{W^{1, \Phi}\left(\mathbb{R}^{n}\right)}=\|f\|_{L^{\Phi}\left(\mathbb{R}^{n}\right)}+c_{0}^{-1} \lim _{\epsilon \rightarrow 0^{+}}\|f\|_{\Phi, \sharp}^{\epsilon}
$$

where $c_{0}$ is the same as in Theorem 1.A.

Applying Theorem 1.10 and Corollary 1.11, we can obtain the following conclusions which are of independent interests.

Corollary 1.12. The conclusions of Theorem 1.10 and Corollary 1.11 hold true if $\Phi$ satisfies one of the following items:

(i) for any $x \in \mathbb{R}^{n}$ and $t \in[0, \infty)$, $\Phi(x, t):=t^{p(x)}$, where $p(\cdot)$ is as in Theorem 1.A.

(ii) for any $x \in \mathbb{R}^{n}$ and $t \in[0, \infty), \Phi(x, t):=\varphi(t)$, where $\varphi$ is an Orlicz function satisfying both the $\Delta_{2}$-condition and that there exist positive constants $l \in(1, \infty)$ and $t_{0} \in[0, \infty)$ such that, for any $t \in\left[t_{0}, \infty\right)$,

$$
\varphi(l t) \geq 2 l \varphi(t)
$$

(iii) for any $x \in \mathbb{R}^{n}$ and $t \in[0, \infty), \Phi(x, t):=\omega(x) t^{p}$, where $p \in(1, \infty)$ and $\omega \in A_{p}\left(\mathbb{R}^{n}\right)$.

(iv) for any $x \in \mathbb{R}^{n}$ and $t \in[0, \infty)$, $\Phi(x, t):=t^{p}+\omega(x) t^{q}$, where $1<p<q<\infty$ and $\omega \in A_{q}\left(\mathbb{R}^{n}\right)$.

The proof of Corollary 1.12 is also presented in Section 2.

Remark 1.13. (i) We point out that Corollary 1.12(i) improves Theorem 1.A via weakening the assumption $f \in L^{1}\left(\mathbb{R}^{n}\right)$ into $f \in L_{\mathrm{loc}}^{1}\left(\mathbb{R}^{n}\right)$, which positively confirms a conjecture proposed by Hästö and Ribeiro in [25, Remark 4.1] (see also Remark 1.4).

(ii) The condition (1.8) in Corollary 1.12(ii) is to guarantee that the complementary function $\varphi^{*}$ to $\varphi$ satisfies the $\Delta_{2}$-condition. A typical example of such a $\varphi$ is that, for any $t \in[0, \infty)$, $\varphi(t):=t^{p}(|\log t|+1)$, where $p \in(1, \infty)$ is a positive constant (see, for example, [27, p. 27, (4.13)]).

(iii) We point out that Musielak-Orlicz functions $\Phi$ as in Corollary 1.12(iv) and the corresponding Musielak-Orlicz-Sobolev spaces naturally appear in the study on the double phase variational problems (see, for example, $[4,10]$ ). 
Remark 1.14. Some time later after we have completed the first version of this article, we learned from Professor Peter Hästö that a result similar to Theorem 1.10 had been independently obtained by Ferreira et al. [18] for the homogeneous Musielak-Orlicz-Sobolev spaces $\dot{W}^{1, \Phi}\left(\mathbb{R}^{n}\right)$ and $\dot{W}^{1, \Phi}(\Omega)$ under the quite different assumptions for $\Phi$, where $\Omega$ is an open set in $\mathbb{R}^{n}$.

More precisely, let $\varphi:[0, \infty] \rightarrow[0, \infty]$ be an increasing function. Denote by $\varphi^{-1}:[0, \infty] \rightarrow$ $[0, \infty]$ the left-continuous generalized inverse of $\varphi$, namely, for any $s \in[0, \infty]$,

$$
\varphi^{-1}(s):=\inf \{t \in[0, \infty]: \varphi(t) \geq s\} .
$$

Moreover, a function $g: \mathbb{R} \rightarrow[0, \infty]$ is said to be almost increasing if there exists a positive constant $c$ such that, for any $t_{1}, t_{2} \in \mathbb{R}$ with $t_{1} \leq t_{2}, g\left(t_{1}\right) \leq c g\left(t_{2}\right)$.

Let the Musielak-Orlicz function $\Phi$ satisfy the following assumptions:

(a) $\Phi$ satisfies the $\Delta_{2}$-condition.

(b) There exists a positive constant $\ell \in(1, \infty)$ such that, for almost every $x \in \mathbb{R}^{n}$, the function $s \mapsto s^{-\ell} \Phi(x, s)$ in $(0, \infty)$ is almost increasing with the constant $\ell$ independent of $x$.

(c) There exist positive constants $\beta \in(0,1)$ and $\gamma \in(0, \infty)$ such that

(c) 1 for any $x \in \mathbb{R}^{n}, \Phi(x, \beta \gamma) \leq 1 \leq \Phi(x, \gamma)$;

(c) 2 for any ball $B \subset \mathbb{R}^{n}$, any $x, y \in B$ and $t \in\left[\gamma, \Phi^{-1}\left(y,|B|^{-1}\right)\right], \Phi(x, \beta t) \leq \Phi(y, t)$;

(c) 3 there exists a function $h \in L^{1}\left(\mathbb{R}^{n}\right) \cap L^{\infty}\left(\mathbb{R}^{n}\right)$ satisfying that, for almost every $x, y \in \mathbb{R}^{n}$ and any $t \in[0, \gamma]$,

$$
\Phi(x, \beta t) \leq \Phi(y, t)+h(x)+h(y) .
$$

For such a Musielak-Orlicz function $\Phi$, the conclusion of Theorem 1.10 was established in [18, Theorem 1.1]. Moreover, replaced $\mathbb{R}^{n}$ by an open set $\Omega \subset \mathbb{R}^{n}$, the conclusion of Theorem 1.10 was also obtained in [18, Theorem 1.1] under the assumptions (a), (b) and (c) with replacing $\mathbb{R}^{n}$ by $\Omega$.

We point out that, in the case of the Euclidean space $\mathbb{R}^{n}$, Theorem 1.10 completely covers $[18$, Theorem 1.1]; in other words, the assumptions in Theorem 1.10 are weaker than the assumptions of [18, Theorem 1.1]. Indeed, by [24, Theorem 4.6], we know that the above assumptions (b) and (c) imply Assumption 1.8. Moreover, from [22, Theorem 6.5] and [24, Theorem 4.6], it follows that the above assumptions (a), (b) and (c) imply Assumption 1.7. Furthermore, it is easy to see that the above assumptions (a) and (c) 1 imply Assumption 1.5. By [23, Lemma 2.4(2)] and [22, Lemma 2.4], we conclude that the above assumptions (a) and (b) imply that the complementary function $\Phi^{*}$ to $\Phi$ satisfies Assumption 1.6, which, combined with [22, Proposition 4.6] and the above assumptions (a) and (c) $)_{1}$, further implies that the complementary function $\Phi^{*}$ satisfies Assumption 1.5. Thus, the above assumptions (a), (b) and (c) for $\Phi$ imply that Assumptions 1.5 through 1.8 hold true for $\Phi$ and that the complementary function $\Phi^{*}$ to $\Phi$ satisfies Assumptions 1.5 and 1.6, which further implies that Theorem 1.10 completely covers [18, Theorem 1.1] in the case of $\mathbb{R}^{n}$.

We point out that (i) and (ii) of Corollary 1.12 are also simple corollaries of [18, Theorem 1.1]. However, the function $\Phi$ as in (iii) and (iv) of Corollary 1.12 may not satisfy the assumption (c) $)_{1}$. Thus, (iii) and (iv) of Corollary 1.12 cannot be deduced from [18, Theorem 1.1], but they can be deduced from Theorem 1.10. In this sense, Theorem 1.10 strictly has more generality than [18, Theorem 1.1] in the case of the Euclidean space $\mathbb{R}^{n}$. 
Finally, we make some conventions on notation. Throughout the article, we always denote by $C$ a positive constant which is independent of the main parameters, but it may vary from line to line. We also use $C_{(\gamma, \beta, \ldots)}$ to denote a positive constant depending on the indicated parameters $\gamma, \beta$, $\ldots$. The symbol $f \lesssim g$ means that $f \leq C g$. If $f \lesssim g$ and $g \lesssim f$, then we write $f \sim g$. We also use the following convention: If $f \leq C g$ and $g=h$ or $g \leq h$, we then write $f \lesssim g \sim h$ or $f \lesssim g \lesssim h$, rather than $f \lesssim g=h$ or $f \lesssim g \leq h$. For any measurable subset $E$ of $\mathbb{R}^{n}$, we denote by $\mathbf{1}_{E}$ its characteristic function. We also let $\mathbb{N}:=\{1,2, \ldots\}$ and use $\overrightarrow{0}_{n}$ to denote the origin of $\mathbb{R}^{n}$.

\section{Proofs of Theorem 1.10 and Corollary 1.12}

In this section, we give the proofs of Theorem 1.10 and Corollary 1.12. We begin with some auxiliary conclusions. In what follows, we use the symbol $C_{c}^{2}\left(\mathbb{R}^{n}\right)$ to denote the set of all functions having continuous derivatives till order 2 with compact supports.

Lemma 2.1. Assume that the Musielak-Orlicz function $\Phi$ satisfies Assumptions 1.5 and 1.6. If $f \in C_{c}^{2}\left(\mathbb{R}^{n}\right)$, then

$$
\lim _{\epsilon \rightarrow 0^{+}} \rho_{\sharp}^{\epsilon}(f)=\rho_{\Phi}\left(c_{0}|\nabla f|\right),
$$

where $c_{0}$ is the same as in Theorem 1.A.

To prove Lemma 2.1, we need the following properties of Musielak-Orlicz functions.

Lemma 2.2. Assume that the Musielak-Orlicz function $\Phi$ satisfies Assumption 1.6.

(i) For almost every $x \in \mathbb{R}^{n}$, any $a \in(0, \infty)$ and any $b \in(0,1)$, $\Phi(x, a b) \leq b \Phi(x, a)$.

(ii) There exists a positive constant $\gamma \in(\kappa, \infty)$ such that, for almost every $x \in \mathbb{R}^{n}$, any $a \in(0, \infty)$ and any $b \in[1, \infty), \Phi(x, a b) \leq b^{\gamma} \Phi(x, a)$, where $\kappa$ is as in Assumption 1.6.

(iii) For any $\delta \in(0, \infty)$, there exists a positive constant $C_{(\delta)}$, depending on $\delta$, such that, for almost every $x \in \mathbb{R}^{n}$ and any $a, b \in(0, \infty)$,

$$
\Phi(x, a+b) \leq(1+\delta)^{\gamma} \Phi(x, a)+C_{(\delta)} \Phi(x, b)
$$

where $\gamma$ is as in Lemma 2.2(ii).

Proof. We first show (i). By the fact that, for almost every $x \in \mathbb{R}^{n}, \Phi(x, \cdot)$ is convex and $\Phi(x, 0)=$ 0 , we find that, for almost every $x \in \mathbb{R}^{n}$, any $a \in(0, \infty)$ and any $b \in(0,1), \Phi(x, a b) \leq b \Phi(x, a)$, which implies that (i) holds true.

Now we give the proof of (ii). From the proof of [23, Lemma 2.4(2)], it follows that there exists a positive constant $\gamma \in(\kappa, \infty)$ such that, for almost every $x \in \mathbb{R}^{n}$, the function $t \mapsto t^{-\gamma} \Phi(x, t)$ is decreasing in $(0, \infty)$, which further implies that, for almost every $x \in \mathbb{R}^{n}$, any $a \in(0, \infty)$ and any $b \in[1, \infty),(a b)^{-\gamma} \Phi(x, a b) \leq a^{-\gamma} \Phi(x, a)$. By this, we find that, for almost every $x \in \mathbb{R}^{n}$, any $a \in(0, \infty)$ and any $b \in[1, \infty)$,

$$
\Phi(x, a b) \leq b^{\gamma} \Phi(x, a) .
$$


Finally, we prove (iii). Let $\delta \in(0, \infty)$. Fix $x \in \mathbb{R}^{n}$ and $a, b \in[0, \infty)$. If $b>\delta a$, from the fact that $\Phi(x, \cdot)$ is increasing and Assumption 1.6, we deduce that

$$
\Phi(x, a+b) \leq \Phi\left(x, b\left(1+\frac{1}{\delta}\right)\right) \leq \Phi\left(x, 2^{m} b\right) \leq \kappa^{m} \Phi(x, b),
$$

where $\kappa$ is as in Assumption 1.6 and $m:=m(\delta) \in \mathbb{N}$ satisfies that $1+\frac{1}{\delta} \leq 2^{m}$. Moreover, if $b \leq \delta a$, by (ii), we conclude that

$$
\Phi(x, a+b) \leq \Phi(x, a(1+\delta)) \leq(1+\delta)^{\gamma} \Phi(x, a)
$$

which, combined with (2.1), then completes the proof of (iii) and hence of Lemma 2.2.

Now we show Lemma 2.1 by using Lemma 2.2 .

Proof of Lemma 2.1. Let $f \in C_{c}^{2}\left(\mathbb{R}^{n}\right)$. Then, by the Taylor expansion, we know that, for any $x, y \in \mathbb{R}^{n}$,

$$
f(y)=f(x)+\nabla f(x) \cdot(y-x)+R(x, y),
$$

where $R(x, y)=o(|x-y|)$ as $y \rightarrow x$, which implies that, for any $x, y \in \mathbb{R}^{n}$ and $r \in(0, \infty)$,

$$
\begin{aligned}
f(y) & -f_{B(x, r)} \\
= & \frac{1}{|B(x, r)|} \int_{B(x, r)}[f(y)-f(z)] d z \\
= & \frac{1}{|B(x, r)|} \int_{B(x, r)}[\nabla f(x) \cdot(y-x)-\nabla f(x) \cdot(z-x)+R(x, y)-R(x, z)] d z .
\end{aligned}
$$

From symmetry, it follows that

$$
\int_{B(x, r)} \nabla f(x) \cdot(z-x) d z=0
$$

which, together with (2.2), further implies that, for any $x, y \in \mathbb{R}^{n}$ and $r \in(0, \infty)$,

$$
f(y)-f_{B(x, r)}=\nabla f(x) \cdot(y-x)+R(x, y)-\frac{1}{|B(x, r)|} \int_{B(x, r)} R(x, z) d z .
$$

By (2.3) and the triangle inequality on $\mathbb{C}$, we conclude that, for any $x, y \in \mathbb{R}^{n}$ and $r \in(0, \infty)$,

$$
\begin{aligned}
M_{B(x, r)}^{\sharp}(f) & \leq \frac{1}{|B(x, r)|} \int_{B(x, r)}|\nabla f(x) \cdot(y-x)| d y+\frac{2}{|B(x, r)|} \int_{B(x, r)}|R(x, y)| d y \\
& =\frac{|\nabla f(x)|}{|B(x, r)|} \int_{B(x, r)}\left|\frac{\nabla f(x)}{|\nabla f(x)|} \cdot(y-x)\right| d y+\frac{2}{|B(x, r)|} \int_{B(x, r)}|R(x, y)| d y .
\end{aligned}
$$

Noticing that $\int_{B(x, r)}|v \cdot(y-x)| d y$ is independent of $v \in S^{n-1}$, from (2.4), we further deduce that, for any $x, y \in \mathbb{R}^{n}$ and $r \in(0, \infty)$,

$$
M_{B(x, r)}^{\sharp}(f) \leq c_{0} r|\nabla f(x)|+\frac{2}{|B(x, r)|} \int_{B(x, r)}|R(x, y)| d y,
$$


where $c_{0}$ is the same as in Theorem 1.A. Similarly to (2.5), we also have

$$
M_{B(x, r)}^{\sharp}(f) \geq c_{0} r|\nabla f(x)|-\frac{2}{|B(x, r)|} \int_{B(x, r)}|R(x, y)| d y .
$$

Let $\Omega:=\left\{x \in \mathbb{R}^{n}:\left.f\right|_{B(x, 1)} \not \equiv 0\right\}$. Then

$$
\rho_{\sharp}^{\epsilon}(f)=\int_{0}^{1}\left[\int_{\Omega} \Phi\left(x, \frac{1}{r} M_{B(x, r)}^{\sharp}(f)\right) d x\right] \psi_{\epsilon}(r) d r .
$$

By $f \in C_{c}^{2}\left(\mathbb{R}^{n}\right)$, we know that $\Omega$ is a bounded set in $\mathbb{R}^{n}$. From (2.5), (2.6) and the fact that $M_{B(x, r)}^{\sharp}(f) \geq 0$, it follows that

$$
\begin{aligned}
& \int_{0}^{1}\left\{\int_{\Omega} \Phi\left(x, \max \left\{0, c_{0}|\nabla f(x)|-h(x, r)\right\}\right) d x\right\} \psi_{\epsilon}(r) d r \\
& \quad \leq \rho_{\sharp}^{\epsilon}(f) \leq \int_{0}^{1}\left\{\int_{\Omega} \Phi\left(x, c_{0}|\nabla f(x)|+h(x, r)\right) d x\right\} \psi_{\epsilon}(r) d r,
\end{aligned}
$$

where $h(x, r):=\frac{2}{r} \frac{1}{|B(x, r)|} \int_{B(x, r)}|R(x, y)| d y$.

By Lemma 2.2(iii), we conclude that, for any $\delta \in(0, \infty)$, there exists a positive constant $C_{(\delta)}$, depending on $\delta$, such that, for almost every $x \in \mathbb{R}^{n}$,

$$
\Phi\left(x, c_{0}|\nabla f(x)|+h(x, r)\right) \leq(1+\delta)^{\gamma} \Phi\left(x, c_{0}|\nabla f(x)|\right)+C_{(\delta)} \Phi(x, h(x, r)),
$$

which, together with $\int_{0}^{1} \psi_{\epsilon}(r) d r=1$, further implies that

$$
\rho_{\sharp}^{\epsilon}(f) \leq(1+\delta)^{\gamma} \int_{\Omega} \Phi\left(x, c_{0}|\nabla f(x)|\right) d x+C_{(\delta)} \int_{0}^{1}\left[\int_{\Omega} \Phi(x, h(x, r)) d x\right] \psi_{\epsilon}(r) d r .
$$

Letting $\epsilon \rightarrow 0^{+}$in (2.8), we find that

$$
\limsup _{\epsilon \rightarrow 0^{+}} \rho_{\sharp}^{\epsilon}(f) \leq(1+\delta)^{\gamma} \rho_{\Phi}\left(c_{0}|\nabla f|\right)+\limsup _{\epsilon \rightarrow 0^{+}} C_{(\delta)} \int_{0}^{1}\left[\int_{\Omega} \Phi(x, h(x, r)) d x\right] \psi_{\epsilon}(r) d r .
$$

Assume that $\epsilon \in(0,1 / 2)$. Let $\sigma \in(0, \infty)$ be such that $\frac{|R(x, y)|}{|x-y|}<\epsilon$ when $|x-y|<\sigma$. By $f \in C_{c}^{2}\left(\mathbb{R}^{n}\right)$, we know that $\frac{|R(x, y)|}{|x-y|}$ is bounded, which further implies that there exists a positive constant $C$ such that

$$
\begin{aligned}
h(x, r) & =\frac{2}{r} \frac{1}{|B(x, r)|} \int_{B(x, r)}|R(x, y)| d y \\
& \leq \frac{2}{|B(x, r)|} \int_{B(x, r)} \frac{|R(x, y)|}{|x-y|} d y \leq 2 \epsilon \mathbf{1}_{(0, \sigma)}(r)+C \mathbf{1}_{(\sigma, 1)}(r) .
\end{aligned}
$$

From this, the definition of $\psi_{\epsilon}$ and Lemma 2.2(i), we deduce that

$$
\int_{0}^{1}\left[\int_{\Omega} \Phi(x, h(x, r)) d x\right] \psi_{\epsilon}(r) d r
$$




$$
\begin{aligned}
& \leq \int_{\Omega}\left[\int_{0}^{\sigma} \Phi(x, 2 \epsilon) \psi_{\epsilon}(r) d r+\int_{\sigma}^{1} \Phi(x, C) \psi_{\epsilon}(r) d r\right] d x \\
& \leq 2 \epsilon \int_{\Omega} \Phi(x, 1) d x+\int_{\Omega} \Phi(x, C) d x \int_{\sigma}^{1} \psi_{\epsilon}(r) d r,
\end{aligned}
$$

which, combined with Assumption 1.5 and the definition of $\psi_{\epsilon}$, further implies that

$$
\lim _{\epsilon \rightarrow 0^{+}} \int_{0}^{1}\left[\int_{\Omega} \Phi(x, h(x, r)) d x\right] \psi_{\epsilon}(r) d r=0 .
$$

By this and (2.9), we conclude that

$$
\limsup _{\epsilon \rightarrow 0^{+}} \rho_{\sharp}^{\epsilon}(f) \leq(1+\delta)^{\gamma} \rho_{\Phi}\left(c_{0}|\nabla f|\right) .
$$

Letting $\delta \rightarrow 0^{+}$in (2.11), then we know that

$$
\limsup _{\epsilon \rightarrow 0^{+}} \rho_{\sharp}^{\epsilon}(f) \leq \rho_{\Phi}\left(c_{0}|\nabla f|\right) .
$$

Moreover, from Lemma 2.2(iii), we deduce that, for any $\delta \in(0, \infty)$, there exists a positive constant $C_{(\delta)}$, depending on $\delta$, such that, for almost every $x \in \mathbb{R}^{n}$ and any $r \in(0, \infty)$,

$$
\begin{aligned}
& \Phi\left(x, \max \left\{0, c_{0}|\nabla f(x)|-h(x, r)\right\}\right) \\
& \quad \geq(1+\delta)^{-\gamma} \Phi\left(x, c_{0}|\nabla f(x)|\right)-C_{(\delta)}(1+\delta)^{-\gamma} \Phi(x, h(x, r)),
\end{aligned}
$$

which, together with (2.7), implies that

$$
\begin{aligned}
& \liminf _{\epsilon \rightarrow 0^{+}} \rho_{\sharp}^{\epsilon}(f) \\
& \quad \geq(1+\delta)^{-\gamma} \rho_{\Phi}\left(c_{0}|\nabla f|\right)-\limsup _{\epsilon \rightarrow 0^{+}} C_{(\delta)}(1+\delta)^{-\gamma} \int_{0}^{1}\left[\int_{\Omega} \Phi(x, h(x, r)) d x\right] \psi_{\epsilon}(r) d r .
\end{aligned}
$$

By this and (2.10), similarly to (2.12), we conclude that

$$
\liminf _{\epsilon \rightarrow 0^{+}} \rho_{\sharp}^{\epsilon}(f) \geq \rho_{\Phi}\left(c_{0}|\nabla f|\right),
$$

which, combined with (2.12), further implies that

$$
\lim _{\epsilon \rightarrow 0^{+}} \rho_{\sharp}^{\epsilon}(f)=\rho_{\Phi}\left(c_{0}|\nabla f|\right) .
$$

This finishes the proof of Lemma 2.1.

Lemma 2.3. Assume that the Musielak-Orlicz function $\Phi$ satisfies Assumptions 1.6 and 1.8. Then there exists a positive constant $C$ such that, for any $\epsilon \in(0, \infty)$ and $f \in \dot{W}^{1, \Phi}\left(\mathbb{R}^{n}\right)$,

$$
\rho_{\sharp}^{\epsilon}(f) \leq C \max \left\{\|\nabla f\|_{L^{\Phi}\left(\mathbb{R}^{n}\right)},\|\nabla f\|_{L^{\Phi}\left(\mathbb{R}^{n}\right)}^{\gamma}\right\},
$$

where $\gamma$ is as in Lemma 2.2(ii). 
Proof. First, let $f \in \dot{W}^{1, \Phi}\left(\mathbb{R}^{n}\right)$ be such that $\|\nabla f\|_{L^{\Phi}\left(\mathbb{R}^{n}\right)} \leq 1$. By the Poincaré inequality, we know that, for any $x \in \mathbb{R}^{n}$ and $r \in(0, \infty)$,

$$
M_{B(x, r)}^{\sharp}(f)=\frac{1}{|B(x, r)|} \int_{B(x, r)}\left|f(y)-f_{B(x, r)}\right| d y \lesssim \frac{r}{|B(x, r)|} \int_{B(x, r)}|\nabla f(y)| d y,
$$

which, together with the fact that, for any $\epsilon \in(0, \infty), \int_{0}^{1} \psi_{\epsilon}(r) d r=1$, further implies that

$$
\begin{aligned}
\rho_{\sharp}^{\epsilon}(f) & \lesssim \int_{0}^{1}\left[\int_{\mathbb{R}^{n}} \Phi\left(x, \frac{1}{|B(x, r)|} \int_{B(x, r)}|\nabla f(y)| d y\right) d x\right] \psi_{\epsilon}(r) d r \\
& \lesssim \int_{0}^{1}\left[\int_{\mathbb{R}^{n}} \Phi(x, M(|\nabla f|)(x)) d x\right] \psi_{\epsilon}(r) d r \\
& \sim \int_{\mathbb{R}^{n}} \Phi(x, M(|\nabla f|)(x)) d x .
\end{aligned}
$$

Moreover, from (i) and (ii) of Lemma 2.2, Assumption 1.8 and $\|\mid \nabla f\|_{L^{\Phi}\left(\mathbb{R}^{n}\right)} \leq 1$, we deduce that

$$
\begin{aligned}
\int_{\mathbb{R}^{n}} \Phi(x, M(|\nabla f|)(x)) d x & \lesssim \max \left\{\|M(|\nabla f|)\|_{L^{\Phi}\left(\mathbb{R}^{n}\right)},\|M(|\nabla f|)\|_{L^{\Phi}\left(\mathbb{R}^{n}\right)}^{\gamma}\right\} \\
& \lesssim \max \left\{\|\nabla f\|_{L^{\Phi}\left(\mathbb{R}^{n}\right)},\|\mid\| f \|_{L^{\Phi}\left(\mathbb{R}^{n}\right)}^{\gamma}\right\} \lesssim 1,
\end{aligned}
$$

which, combined with (2.13), implies that

$$
\rho_{\sharp}^{\epsilon}(f) \lesssim \int_{\mathbb{R}^{n}} \Phi(x, M(|\nabla f|)(x)) d x \lesssim 1 .
$$

For any $f \in \dot{W}^{1, \Phi}\left(\mathbb{R}^{n}\right)$, replacing $f$ by $f /\|\nabla f\|_{L^{\Phi}\left(\mathbb{R}^{n}\right)}$ and repeating the proof of (2.14), we know that

$$
\rho_{\sharp}^{\epsilon}\left(\frac{f}{\|\nabla f\|_{L^{\Phi}\left(\mathbb{R}^{n}\right)}}\right) \lesssim \int_{\mathbb{R}^{n}} \Phi\left(x, \frac{M(|\nabla f|)(x)}{\|\nabla f \mid\|_{L^{\Phi}\left(\mathbb{R}^{n}\right)}}\right) d x \lesssim 1 .
$$

Moreover, from the definition of $\rho_{\sharp}^{\epsilon}$ and (i) and (ii) of Lemma 2.2, it follows that, for any $\lambda \in(0, \infty)$,

$$
\rho_{\sharp}^{\epsilon}(f) \leq \rho_{\sharp}^{\epsilon}\left(\frac{f}{\lambda}\right) \max \left\{\lambda, \lambda^{\gamma}\right\},
$$

where $\gamma$ is as in Lemma 2.2(ii), which, combined with (2.15), further implies that

$$
\rho_{\sharp}^{\epsilon}(f) \lesssim \max \left\{\|\mid \nabla f\|_{L^{\Phi}\left(\mathbb{R}^{n}\right)},\|\nabla f\|_{L^{\Phi}\left(\mathbb{R}^{n}\right)}^{\gamma}\right\} .
$$

This finishes the proof of Lemma 2.3.

To prove Theorem 1.10, we need the following reflexivity of the Musielak-Orlicz space $L^{\Phi}\left(\mathbb{R}^{n}\right)$, which was obtained in [39, Theorem 1.4]. 
Lemma 2.4. Let $\Phi$ be a Musielak-Orlicz function and $\Phi^{*}$ be the complementary function to $\Phi$. If both $\Phi$ and $\Phi^{*}$ satisfy Assumptions 1.5 and 1.6, then the Musielak-Orlicz space $L^{\Phi}\left(\mathbb{R}^{n}\right)$ is reflexive, namely, $\left(L^{\Phi}\left(\mathbb{R}^{n}\right)\right)^{* *}=L^{\Phi}\left(\mathbb{R}^{n}\right)$, where $\left(L^{\Phi}\left(\mathbb{R}^{n}\right)\right)^{*}$ denotes the dual space of $L^{\Phi}\left(\mathbb{R}^{n}\right)$ [namely, the space of all continuous linear functions on $\left.L^{\Phi}\left(\mathbb{R}^{n}\right)\right]$ and

$$
\left(L^{\Phi}\left(\mathbb{R}^{n}\right)\right)^{* *}=\left(\left(L^{\Phi}\left(\mathbb{R}^{n}\right)\right)^{*}\right)^{*}
$$

Let $G \in C_{c}^{\infty}\left(B\left(\overrightarrow{0}_{n}, 1\right)\right)$ be a standard mollifier. Namely, for any $x \in \mathbb{R}^{n}$,

$$
G(x):= \begin{cases}C_{1} e^{-\frac{1}{1-|x|^{2}}} & \text { if }|x|<1 \\ 0 & \text { if }|x| \geq 1\end{cases}
$$

where $C_{1}$ is a positive constant such that $\int_{\mathbb{R}^{n}} G(x) d x=1$. For any $\varepsilon \in(0, \infty)$ and $x \in \mathbb{R}^{n}$, let $G_{\varepsilon}(x):=\varepsilon^{-n} G(x / \varepsilon)$.

Now we prove Theorem 1.10 by using Lemmas 2.1 through 2.4 .

Proof of Theorem 1.10. We divide the proof into the following three steps according to the sufficient and the necessary conditions for (1.5) and the equivalence of (1.6) and (1.7).

Step 1) In this step, we show that, if $|\nabla f| \in L^{\Phi}\left(\mathbb{R}^{n}\right)$, then (1.6) holds true, which further implies that (1.5) also holds true.

By the triangle inequality on $\mathbb{C}$, we find that, for any $x \in \mathbb{R}^{n}, r \in(0, \infty)$ and $f, g \in L_{\mathrm{loc}}^{1}\left(\mathbb{R}^{n}\right)$,

$$
\begin{aligned}
& \frac{1}{|B(x, r)|} \int_{B(x, r)}\left|f(y)-f_{B(x, r)}\right| d y \\
& \quad \leq \frac{1}{|B(x, r)|} \int_{B(x, r)}\left|f(y)-g(y)-(f-g)_{B(x, r)}\right| d y+\frac{1}{|B(x, r)|} \int_{B(x, r)}\left|g(y)-g_{B(x, r)}\right| d y,
\end{aligned}
$$

which implies that, for any $x \in \mathbb{R}^{n}, r \in(0, \infty)$ and $f, g \in L_{\text {loc }}^{1}\left(\mathbb{R}^{n}\right)$,

$$
M_{B(x, r)}^{\sharp}(f) \leq M_{B(x, r)}^{\sharp}(f-g)+M_{B(x, r)}^{\sharp}(g) .
$$

From this, the definition of $\rho_{\sharp}^{\epsilon}(f)$ and Lemma 2.2(iii), we deduce that, for any $\epsilon \in(0, \infty)$ and $\delta \in(0, \infty)$, there exists a positive constant $C_{(\delta)}$, depending on $\delta$, such that

$$
\rho_{\sharp}^{\epsilon}(f) \leq C_{(\delta)} \rho_{\sharp}^{\epsilon}(f-g)+(1+\delta)^{\gamma} \rho_{\sharp}^{\epsilon}(g),
$$

here and hereafter, $\gamma$ is as in Lemma 2.2(ii).

Let $f \in \dot{W}^{1, \Phi}\left(\mathbb{R}^{n}\right)$ and $g \in C_{c}^{\infty}\left(\mathbb{R}^{n}\right)$. Then, by (2.17), Lemmas 2.1 and 2.3, we conclude that

$$
\limsup _{\epsilon \rightarrow 0^{+}} \rho_{\sharp}^{\epsilon}(f) \leq C_{(\delta)} \max \left\{\||\nabla(f-g)|\|_{L^{\Phi}\left(\mathbb{R}^{n}\right)},\|\mid \nabla(f-g)\|_{L^{\Phi}\left(\mathbb{R}^{n}\right)}^{\gamma}\right\}+(1+\delta)^{\gamma} \rho_{\Phi}\left(c_{0}|\nabla g|\right),
$$

where $c_{0}$ is the same as in Theorem 1.A. From Assumption 1.7, it follows that $C_{c}^{\infty}\left(\mathbb{R}^{n}\right)$ is dense in $\dot{W}^{1, \Phi}\left(\mathbb{R}^{n}\right)$, which implies that there exists a sequence $\left\{g_{i}\right\}_{i \in \mathbb{N}} \subset C_{c}^{\infty}\left(\mathbb{R}^{n}\right)$ such that $\nabla g_{i}$ converges to $\nabla f$ in $L^{\Phi}\left(\mathbb{R}^{n}\right)$ as $i \rightarrow \infty$. Replacing $g$ by $g_{i}$ in (2.18) and letting $i \rightarrow \infty$, we obtain

$$
\limsup _{\epsilon \rightarrow 0^{+}} \rho_{\sharp}^{\epsilon}(f) \leq \lim _{i \rightarrow \infty}(1+\delta)^{\gamma} \rho_{\Phi}\left(c_{0}\left|\nabla g_{i}\right|\right)=(1+\delta)^{\gamma} \rho_{\Phi}\left(c_{0}|\nabla f|\right) .
$$


Letting $\delta \rightarrow 0^{+}$in (2.19), we conclude that

$$
\limsup _{\epsilon \rightarrow 0^{+}} \rho_{\sharp}^{\epsilon}(f) \leq \rho_{\Phi}\left(c_{0}|\nabla f|\right) .
$$

Moreover, similarly to (2.17), we find that, for any $\epsilon \in(0, \infty)$ and $\delta \in(0, \infty)$, there exists a positive constant $C_{(\delta)}$, depending on $\delta$, such that

$$
\rho_{\sharp}^{\epsilon}(f) \geq \frac{1}{(1+\delta)^{\gamma}} \rho_{\sharp}^{\epsilon}\left(g_{i}\right)-\frac{C_{(\delta)}}{(1+\delta)^{\gamma}} \rho_{\sharp}^{\epsilon}\left(f-g_{i}\right),
$$

where, for any $i \in \mathbb{N}, g_{i}$ is as in (2.19). By this estimate and similarly to (2.20), we conclude that

$$
\liminf _{\epsilon \rightarrow 0^{+}} \rho_{\sharp}^{\epsilon}(f) \geq \rho_{\Phi}\left(c_{0}|\nabla f|\right),
$$

which, together with (2.20), further implies that

$$
\lim _{\epsilon \rightarrow 0^{+}} \rho_{\sharp}^{\epsilon}(f)=\rho_{\Phi}\left(c_{0}|\nabla f|\right) .
$$

Thus, (1.6) holds true.

Step 2) In this step, we show that, if (1.5) holds true, then $|\nabla f| \in L^{\Phi}\left(\mathbb{R}^{n}\right)$.

Let $G$ be as in (2.16). By the triangle inequality on $\mathbb{C}$ and a change of integration order, we find that, for any $x \in \mathbb{R}^{n}, r \in(0,1)$ and $\delta \in(0, \infty)$,

$$
\begin{aligned}
M_{B(x, r)}^{\sharp}\left(G_{\delta} * f\right) & =\frac{1}{|B(x, r)|} \int_{B(x, r)}\left|\left(G_{\delta} * f\right)(y)-\left(G_{\delta} * f\right)_{B(x, r)}\right| d y \\
& =\frac{1}{|B(x, r)|} \int_{B(x, r)}\left|G_{\delta} *\left(f-f_{B(\cdot, r)}\right)(y)\right| d y \leq G_{\delta} * M_{B(, r)}^{\sharp}(f)(x) .
\end{aligned}
$$

For any $\delta \in(0, \infty), x \in \mathbb{R}^{n}$ and $r \in(0,1]$, let

$$
h_{r, \delta}(x):=\frac{1}{r} M_{B(x, r)}^{\sharp}\left(G_{\delta} * f\right) \text { and } g_{r}(x):=\frac{1}{r} M_{B(x, r)}^{\sharp}(f) .
$$

If $\left\|h_{r, \delta}\right\|_{L^{\Phi}\left(\mathbb{R}^{n}\right)} \leq 1$, then, from Lemma 2.2(i), it follows that

$$
\int_{\mathbb{R}^{n}} \Phi\left(x, h_{r, \delta}(x)\right) d x=\int_{\mathbb{R}^{n}} \Phi\left(x, \frac{h_{r, \delta}(x)}{\left\|h_{r, \delta}\right\|_{L^{\Phi}\left(\mathbb{R}^{n}\right)}}\left\|h_{r, \delta}\right\|_{L^{\Phi}\left(\mathbb{R}^{n}\right)}\right) d x \leq\left\|h_{r, \delta}\right\|_{L^{\Phi}\left(\mathbb{R}^{n}\right)} \leq 1 .
$$

If $\left\|h_{r, \delta}\right\|_{L^{\Phi}\left(\mathbb{R}^{n}\right)} \in(1, \infty)$, by Lemma 2.2(ii), (2.21) and Assumption 1.8, we find that

$$
\begin{aligned}
\int_{\mathbb{R}^{n}} \Phi\left(x, h_{r, \delta}(x)\right) d x & =\int_{\mathbb{R}^{n}} \Phi\left(x, \frac{h_{r, \delta}(x)}{\left\|h_{r, \delta}\right\|_{L^{\Phi}\left(\mathbb{R}^{n}\right)}}\left\|h_{r, \delta}\right\|_{L^{\Phi}\left(\mathbb{R}^{n}\right)}\right) d x \\
& \leq\left\|h_{r, \delta}\right\|_{L^{\Phi}\left(\mathbb{R}^{n}\right)}^{\gamma} \leq\left\|G_{\delta} * g_{r}\right\|_{L^{\Phi}\left(\mathbb{R}^{n}\right)}^{\gamma} \lesssim\left\|M\left(g_{r}\right)\right\|_{L^{\Phi}\left(\mathbb{R}^{n}\right)}^{\gamma} \lesssim\left\|g_{r}\right\|_{L^{\Phi}\left(\mathbb{R}^{n}\right)}^{\gamma},
\end{aligned}
$$

which, further implies that, if $\left\|h_{r, \delta}\right\|_{L^{\Phi}\left(\mathbb{R}^{n}\right)} \in(1, \infty)$, then $\left\|g_{r}\right\|_{L^{\Phi}\left(\mathbb{R}^{n}\right)} \gtrsim 1$. From this and Lemma 2.2(i), we deduce that

$$
\left\|g_{r}\right\|_{L^{\Phi}\left(\mathbb{R}^{n}\right)} \lesssim \int_{\mathbb{R}^{n}} \Phi\left(x, g_{r}(x)\right) d x .
$$


By this and (2.23), we conclude that, if $\left\|h_{r, \delta}\right\|_{L^{\Phi}\left(\mathbb{R}^{n}\right)} \in(1, \infty)$, then

$$
\int_{\mathbb{R}^{n}} \Phi\left(x, h_{r, \delta}(x)\right) d x \lesssim\left\{\int_{\mathbb{R}^{n}} \Phi\left(x, g_{r}(x)\right) d x\right\}^{\gamma}
$$

which, combined with (2.22) and the fact that, for any $\epsilon \in(0, \infty), \int_{0}^{1} \psi_{\epsilon}(r) d r=1$, further implies that, for any $\delta, \epsilon \in(0, \infty)$,

$$
\begin{aligned}
& \int_{0}^{1}\left\{\int_{\mathbb{R}^{n}} \Phi\left(x, \frac{1}{r} M_{B(x, r)}^{\sharp}\left(G_{\delta} * f\right)\right) d x\right\}^{1 / \gamma} \psi_{\epsilon}(r) d r \\
& \quad \lesssim \int_{0}^{1}\left[\int_{\mathbb{R}^{n}} \Phi\left(x, \frac{1}{r} M_{B(x, r)}^{\sharp}(f)\right) d x+1\right] \psi_{\epsilon}(r) d r \lesssim \rho_{\sharp}^{\epsilon}(f)+1 .
\end{aligned}
$$

Assume that $g \in C^{2}\left(\mathbb{R}^{n}\right)$. Repeating the proof of Lemma 2.1, we know that, for any $R \in(0, \infty)$,

$$
\begin{aligned}
& \lim _{\epsilon \rightarrow 0^{+}} \int_{0}^{1}\left\{\int_{B\left(\overrightarrow{0}_{n}, R\right)} \Phi\left(x, \frac{1}{r} M_{B(x, r)}^{\sharp}(g)\right) d x\right\}^{1 / \gamma} \psi_{\epsilon}(r) d r \\
& \quad=\left\{\int_{B\left(\overrightarrow{0}_{n}, R\right)} \Phi\left(x, c_{0}|\nabla g(x)|\right) d x\right\}^{1 / \gamma} .
\end{aligned}
$$

Assume that $\lim _{\epsilon \rightarrow 0^{+}} \rho_{\sharp}^{\epsilon}(f)<\infty$. Noticing that $G_{\delta} * f \in C^{2}\left(\mathbb{R}^{n}\right)$ for any $\delta \in(0, \infty)$, by (2.24) and (2.25), we conclude that, for any $R, \delta \in(0, \infty)$,

$$
\begin{aligned}
& \left\{\int_{B\left(\overrightarrow{0}_{n}, R\right)} \Phi\left(x, c_{0}\left|\nabla\left(G_{\delta} * f\right)(x)\right|\right) d x\right\}^{1 / \gamma} \\
& \quad=\lim _{\epsilon \rightarrow 0^{+}} \int_{0}^{1}\left\{\int_{B\left(\overrightarrow{0}_{n}, R\right)} \Phi\left(x, \frac{1}{r} M_{B(x, r)}^{\sharp}\left(G_{\delta} * f\right)\right) d x\right\}^{1 / \gamma} \psi_{\epsilon}(r) d r \\
& \quad \leq \lim _{\epsilon \rightarrow 0^{+}} \int_{0}^{1}\left\{\int_{\mathbb{R}^{n}} \Phi\left(x, \frac{1}{r} M_{B(x, r)}^{\sharp}\left(G_{\delta} * f\right)\right) d x\right\}^{1 / \gamma} \psi_{\epsilon}(r) d r \lesssim \lim _{\epsilon \rightarrow 0^{+}} \rho_{\sharp}^{\epsilon}(f)+1<\infty .
\end{aligned}
$$

Therefore, $\left\{\nabla\left(G_{\delta} * f\right)\right\}_{\delta>0}$ is a bounded sequence in $L^{\Phi}\left(\mathbb{R}^{n}\right)$. By this and Lemma 2.4 , combined with the well-known Eberlein-Šmulian theorem, we conclude that there exists a subsequence of $\left\{\nabla\left(G_{\delta} * f\right)\right\}_{\delta>0}$ weakly converging in $L^{\Phi}\left(\mathbb{R}^{n}\right)$ to a function $h \in L^{\Phi}\left(\mathbb{R}^{n}\right)$, which, together with the definition of the derivative and the fact that $G_{\delta} * f$ converges to $f$ in $L_{\text {loc }}^{1}\left(\mathbb{R}^{n}\right)$, implies that $h=\nabla f$. Thus, $|\nabla f| \in L^{\Phi}\left(\mathbb{R}^{n}\right)$.

Step 3) Finally, we show that (1.6) implies (1.7).

Let $\delta \in(0, \infty)$ and

$$
g:=\frac{f}{c_{0}(1+\delta)\left(\|\| \nabla f \|_{L^{\Phi}\left(\mathbb{R}^{n}\right)}+\delta\right)}
$$

where $c_{0}$ is the same as in Theorem 1.A. From (1.6) and Lemma 2.2(i), it follows that

$$
\lim _{\epsilon \rightarrow 0^{+}} \rho_{\sharp}^{\epsilon}(g)=\rho_{\Phi}\left(c_{0}|\nabla g|\right)=\rho_{\Phi}\left(\frac{|\nabla f|}{(1+\delta)\left(|||\nabla f| \|_{L^{\Phi}\left(\mathbb{R}^{n}\right)}+\delta\right)}\right)
$$




$$
\leq \frac{1}{1+\delta} \rho_{\Phi}\left(\frac{|\nabla f|}{\|\nabla f\|_{L^{\Phi}\left(\mathbb{R}^{n}\right)}+\delta}\right) \leq \frac{1}{1+\delta}
$$

Thus, for any sufficiently small $\epsilon \in(0, \infty), \rho_{\sharp}^{\epsilon}(g) \leq 1$, which implies that $\|g\|_{\sharp, \Phi}^{\epsilon} \leq 1$ and hence

$$
\|f\|_{\sharp, \Phi}^{\epsilon} \leq c_{0}(1+\delta)\left(\|\| \nabla f \|_{L^{\Phi}\left(\mathbb{R}^{n}\right)}+\delta\right) .
$$

Letting $\delta \rightarrow 0^{+}$, we obtain

$$
\lim _{\epsilon \rightarrow 0^{+}}\|f\|_{\sharp, \Phi}^{\epsilon} \leq c_{0}\|\| \nabla f \|_{L^{\Phi}\left(\mathbb{R}^{n}\right)} .
$$

Similarly, we also have

$$
\lim _{\epsilon \rightarrow 0^{+}}\|f\|_{\sharp, \Phi}^{\epsilon} \geq c_{0}\|\| \nabla f \|_{L^{\Phi}\left(\mathbb{R}^{n}\right)} .
$$

Therefore, (1.7) holds true. This finishes the proof of Theorem 1.10.

Now we show Corollary 1.12.

Proof of Corollary 1.12. To prove Corollary 1.12, we only need to show that the Musielak-Orlicz function $\Phi$ as in Corollary 1.12 satisfies Assumptions 1.5 through 1.8 and its complementary function $\Phi^{*}$ satisfies Assumptions 1.5 and 1.6. We divide the proof into the following four steps according to the type of $\Phi$.

Step $i)$ In this step, we show Corollary 1.12(i). To this end, for any $x \in \mathbb{R}^{n}$ and $t \in[0, \infty)$, let $\Phi(x, t):=t^{p(x)}$, where $p(\cdot)$ is as in Theorem 1.A. In this case, by the assumption that $p$ satisfies the log-Hölder continuity condition, we easily know that $p^{+}<\infty$ with $p^{+}$as in (1.2), which, together with the assumption $p^{-} \in(1, \infty)$ with $p^{-}$as in $(1.2)$, further implies that $1<p^{-} \leq p^{+}<\infty$. From this, we deduce that $\Phi$ satisfies Assumptions 1.5 and 1.6. Moreover, it is known that Assumption 1.7 holds true for such a function $\Phi$ (see, for example, [14, Theorem 9.1.6]). Furthermore, from [14, Theorem 4.3.8] (see also [12, 13, 15, 24]), it follows that the Hardy-Littlewood maximal operator $M$ is bounded on $L^{p(\cdot)}\left(\mathbb{R}^{n}\right)$, which further implies that $\Phi$ satisfies Assumption 1.8. Thus, Assumptions 1.5 through 1.8 hold true for such a $\Phi$.

Moreover, it is easy to see that, for any $x \in \mathbb{R}^{n}$ and $t \in[0, \infty)$,

$$
\Phi^{*}(x, t)=\frac{1}{q(x)[p(x)]^{q(x) / p(x)}} t^{q(x)}
$$

where, for any $x \in \mathbb{R}^{n}, q(x)$ is given by the equality $\frac{1}{p(x)}+\frac{1}{q(x)}=1$. Indeed, by the Young inequality, we know that, for any $k \in(1, \infty)$ and $a, b \in[0, \infty)$,

$$
a b \leq \frac{a^{k}}{k}+\frac{b^{k^{\prime}}}{k^{\prime}}
$$

where $k^{\prime} \in(1, \infty)$ is given by the equality $\frac{1}{k}+\frac{1}{k^{\prime}}=1$, which, combined with choosing $a:=$ $s[p(x)]^{1 / p(x)}, b:=t /[p(x)]^{1 / p(x)}$ and $k:=p(x)$ in (2.27), further implies that, for any $x \in \mathbb{R}^{n}$ and $s, t \in[0, \infty)$,

$$
s t \leq s^{p(x)}+\frac{1}{q(x)[p(x)]^{q(x) / p(x)}} t^{q(x)} .
$$


From this and the definition of $\Phi^{*}$, it follows that, for any $x \in \mathbb{R}^{n}$ and $t \in[0, \infty)$,

$$
\Phi^{*}(x, t) \leq \frac{1}{q(x)[p(x)]^{q(x) / p(x)}} t^{q(x)} .
$$

Furthermore, by the condition $a^{k}=b^{k^{\prime}}$ that is to guarantee that the equality holds true in (2.27), we conclude that, for any $x \in \mathbb{R}^{n}$ and $t \in[0, \infty)$,

$$
\Phi^{*}(x, t) \geq \frac{1}{q(x)[p(x)]^{q(x) / p(x)}} t^{q(x)},
$$

which, combined with (2.28), further implies that (2.26) holds true for any $x \in \mathbb{R}^{n}$ and $t \in[0, \infty)$. From the fact that $1<p^{-} \leq p^{+}<\infty$, we deduce that $1<q^{-} \leq q^{+}<\infty$, which further implies that $\Phi^{*}$ satisfies Assumptions 1.5 and 1.6. Therefore, the conclusions of Theorem 1.10 and Corollary 1.11 hold true for such a $\Phi$. This finishes the proof of Corollary 1.12(i).

Step ii) In this step, we show Corollary 1.12(ii). To this end, for any $x \in \mathbb{R}^{n}$ and $t \in[0, \infty)$, let $\Phi(x, t):=\varphi(t)$, where $\varphi$ is an Orlicz function satisfying the $\Delta_{2}$-condition and (1.8). Obviously, such a $\Phi$ satisfies Assumptions 1.5 and 1.6. It is well known that $C_{c}^{\infty}\left(\mathbb{R}^{n}\right)$ is dense in the homogeneous Orlicz-Sobolev space $\dot{W}^{1, \varphi}\left(\mathbb{R}^{n}\right)$ (see, for example, [3, Theorem 8.31]), which implies that the function $\Phi$ satisfies Assumption 1.7. From [20, Theorem 2.1] (see also [26, Theorem 1.2.1]), we deduce that the Hardy-Littlewood maximal operator $M$ is bounded on $L^{\varphi}\left(\mathbb{R}^{n}\right)$, which implies that such a $\Phi$ satisfies Assumption 1.8. Thus, Assumptions 1.5 through 1.8 hold true for such a $\Phi$.

Furthermore, by the fact that the complementary function $\Phi^{*}$ is independent of the spatial variable $x$, we conclude that Assumption 1.5 holds true for $\Phi^{*}$. From (1.8) and [27, p. 25, Theorem 4.2], it follows that $\Phi^{*}$ satisfies the $\Delta_{2}$-condition. Therefore, Theorem 1.10 and Corollary 1.11 hold true for such a $\Phi$. which completes the proof of Corollary 1.12(ii).

Step iii) In this step, we show Corollary 1.12(iii). To this end, for any $x \in \mathbb{R}^{n}$ and $t \in[0, \infty)$, let $\Phi(x, t):=\omega(x) t^{p}$, where $p \in(1, \infty)$ and $\omega \in A_{p}\left(\mathbb{R}^{n}\right)$. In this case, the Musielak-Orlicz space $L^{\Phi}\left(\mathbb{R}^{n}\right)$ and the homogeneous Musielak-Orlicz-Sobolev space $\dot{W}^{1, \Phi}\left(\mathbb{R}^{n}\right)$ are just the weighted Lebesgue space $L_{\omega}^{p}\left(\mathbb{R}^{n}\right)$ and the homogeneous weighted Sobolev space $\dot{W}_{\omega}^{1, p}\left(\mathbb{R}^{n}\right)$, respectively. Recall that the weighted Lebesgue space $L_{\omega}^{p}\left(\mathbb{R}^{n}\right)$ is defined to be the space of all Lebesgue measurable functions $f$ such that

$$
\|f\|_{L_{\omega}^{p}\left(\mathbb{R}^{n}\right)}:=\left[\int_{\mathbb{R}^{n}}|f(x)| \omega(x) d x\right]^{1 / p}<\infty
$$

and the homogeneous weighted Sobolev space $\dot{W}_{\omega}^{1, p}\left(\mathbb{R}^{n}\right)$ is defined to be the space of all $f \in$ $L_{\text {loc }}^{1}\left(\mathbb{R}^{n}\right)$ such that $|\nabla f| \in L_{\omega}^{p}\left(\mathbb{R}^{n}\right)$. It is easy to see that $\Phi$ satisfies Assumptions 1.5 and 1.6. Moreover, from [37, Theorem 2.1.4], we deduce that $C_{c}^{\infty}\left(\mathbb{R}^{n}\right)$ is dense in the homogeneous weighted Sobolev space $\dot{W}_{\omega}^{1, p}\left(\mathbb{R}^{n}\right)$, which implies that Assumption 1.7 holds true for $\Phi$. Furthermore, it is well known that the Hardy-Littlewood maximal operator $M$ is bounded on the weighted space $L_{\omega}^{p}\left(\mathbb{R}^{n}\right)$ (see, for example, [21, Theorem 7.1.9]), which further implies that $\Phi$ satisfies Assumption 1.8. Thus, Assumptions 1.5 through 1.8 hold true for such a $\Phi$.

Moreover, similarly to (2.26), we find that, for any $x \in \mathbb{R}^{n}$ and $t \in[0, \infty)$,

$$
\Phi^{*}(x, t)=\frac{1}{p^{\prime}} p^{-\frac{p^{\prime}}{p}}[\omega(x)]^{-\frac{p^{\prime}}{p}} t^{p^{\prime}}
$$


where $p^{\prime} \in(1, \infty)$ is given by the equality $\frac{1}{p}+\frac{1}{p^{\prime}}=1$. It is easy to see that Assumption 1.6 holds true for $\Phi^{*}$. Furthermore, from the fact that $\omega \in A_{p}\left(\mathbb{R}^{n}\right)$ and the properties of $A_{p}\left(\mathbb{R}^{n}\right)$-weights, we deduce that $\omega^{-\frac{p^{\prime}}{p}} \in A_{p^{\prime}}\left(\mathbb{R}^{n}\right)$ (see, for example, [21, Proposition 7.1.5(4)]), which implies that $\omega^{-\frac{p^{\prime}}{p}} \in L_{\text {loc }}^{1}\left(\mathbb{R}^{n}\right)$ and hence $\Phi^{*}$ satisfies Assumption 1.5. Therefore, the conclusions of Theorem 1.10 and Corollary 1.11 hold true for such a $\Phi$. This finishes the proof of Corollary 1.12(iii).

Step iv) In this step, we show Corollary 1.12(iv). To this end, for any $x \in \mathbb{R}^{n}$ and $t \in[0, \infty)$, let $\Phi(x, t):=t^{p}+\omega(x) t^{q}$, where $1<p<q<\infty$ and $\omega \in A_{q}\left(\mathbb{R}^{n}\right)$. Then it is easy to see that such a $\Phi$ satisfies Assumptions 1.5 and 1.6. Moreover, by [4, Theorem 1.1], we find that Assumption 1.7 holds true for $\Phi$. From the facts that the Hardy-Littlewood maximal operator $M$ is bounded on both $L^{p}\left(\mathbb{R}^{n}\right)$ and $L_{\omega}^{q}\left(\mathbb{R}^{n}\right)$ (see, for example, [21, Theorem 7.1.9]), it follows that $\Phi$ satisfies Assumption 1.8. Thus, Assumptions 1.5 through 1.8 hold true for such a $\Phi$.

Moreover, similarly to (2.26), we know that, for any $x \in \mathbb{R}^{n}$ and $t \in[0, \infty)$,

$$
\Phi^{*}(x, t) \sim \frac{1}{p^{\prime}} p^{-\frac{p^{\prime}}{p}} t^{p^{\prime}}+\frac{1}{q^{\prime}} q^{-\frac{q^{\prime}}{q}}[\omega(x)]^{-\frac{q^{\prime}}{q}} t^{q^{\prime}},
$$

where $p^{\prime}, q^{\prime} \in(1, \infty)$ are given, respectively, by $\frac{1}{p}+\frac{1}{p^{\prime}}=1$ and $\frac{1}{q}+\frac{1}{q^{\prime}}=1$, and the implicit positive equivalence constants are independent of $x$ and $t$. By this and similarly to the corresponding proof in Step iii), we conclude that both Assumptions 1.5 and 1.6 hold true for $\Phi^{*}$. Therefore, Theorem 1.10 and Corollary 1.11 hold true for such a $\Phi$, which completes the proof of Corollary 1.12(iv) and hence of Corollary 1.12.

\section{References}

[1] E. Acerbi and G. Mingione, Gradient estimates for the $p(x)$-Laplacean system, J. Reine Angew. Math. 584 (2005), 117-148.

[2] E. Acerbi and G. Mingione, Regularity results for a class of functionals with non-standard growth, Arch. Ration. Mech. Anal. 156 (2001), 121-140.

[3] R. A. Adams and J. J. F. Fournier, Sobolev Spaces, Second edition, Pure and Applied Mathematics, 140, Elsevier/Academic Press, Amsterdam, 2003.

[4] Y. Ahmida, P. Gwiazda, I. Skrzypczak and A. Youssfi, Gossez's approximation theorems in the Musielak-Orlicz-Sobolev spaces, J. Funct. Anal. 275 (2018), 2538-2571.

[5] Z. Birnbaum and W. Orlicz, Über die verallgemeinerung des begriffes der zueinander konjugierten potenzen, Studia Math. 3 (1931), 1-67.

[6] J. Bourgain, H. Brezis and P. Mironescu, Another look at Sobolev spaces, in Optimal Control and Partial Differential Equations, 439-455, IOS, Amsterdam, 2001.

[7] J. Bourgain, H. Brezis and P. Mironescu, Limiting embedding theorems for $W^{s, p}$ when $s \uparrow 1$ and applications, J. Anal. Math. 87 (2002), 77-101.

[8] H. Brezis, How to recognize constant functions. A connection with Sobolev spaces, Russian Math. Surveys 57 (2002), 693-708.

[9] M. Colombo and G. Mingione, Calderón-Zygmund estimates and non-uniformly elliptic operators, J. Funct. Anal. 270 (2016), 1416-1478.

[10] M. Colombo and G. Mingione, Bounded minimisers of double phase variational integrals, Arch. Ration. Mech. Anal. 218 (2015), 219-273. 
[11] D. V. Cruz-Uribe and A. Fiorenza, Variable Lebesgue Spaces, Foundations and Harmonic Analysis, Applied and Numerical Harmonic Analysis, Birkhäuser/Springer, Heidelberg, 2013.

[12] L. Diening, Maximal function on generalized Lebesgue spaces $L^{p(\cdot)}$, Math. Inequal. Appl. 7 (2004), 245-253.

[13] L. Diening, Maximal function on Musielak-Orlicz spaces and generalized Lebesgue spaces, Bull. Sci. Math. 129 (2005), 657-700.

[14] L. Diening, P. Harjulehto, P. Hästö and M. Růžička, Lebesgue and Sobolev Spaces with Variable Exponents, Lecture Notes in Mathematics, 2017, Springer, Heidelberg, 2011.

[15] L. Diening, P. Harjulehto, P. Hästö, Y. Mizuta and T. Shimomura, Maximal functions in variable exponent spaces: limiting cases of the exponent, Ann. Acad. Sci. Fenn. Math. 34 (2009), 503-522.

[16] L. Diening and P. Hästö, Variable exponent trace spaces, Studia Math. 183 (2007), 127-141.

[17] J. Fernández Bonder and A. M. Salort, Fractional order Orlicz-Sobolev spaces, arXiv: 1707.03267.

[18] R. Ferreira, P. Hästö and A. M. Ribeiro, Characterization of generalized Orlicz spaces, Commun. Contemp. Math. (to appear) or arXiv: 1612.04566.

[19] A. Fiorenza, A mean continuity type result for certain Sobolev spaces with variable exponent, Commun. Contemp. Math. 4 (2002), 587-605.

[20] D. Gallardo, Orlicz spaces for which the Hardy-Littlewood maximal operator is bounded, Publ. Mat. 32 (1988), 261-266.

[21] L. Grafakos, Classical Fourier Analysis, third edition, Graduate Texts in Mathematics, 249, Springer, New York, 2014.

[22] P. Harjulehto P. Hästö and R. Klén, Generalized Orlicz spaces and related PDE, Nonlinear Anal. 143 (2016), 155-173.

[23] P. Harjulehto, P. Hästö and O. Toivanen, Hölder regularity of quasiminimizers under generalized growth conditions, Calc. Var. Partial Differential Equations 56 (2017), no. 2, Art. 22, $26 \mathrm{pp}$.

[24] P. Hästö, The maximal operator on generalized Orlicz spaces, J. Funct. Anal. 269 (2015), 4038-4048.

[25] P. Hästö and A. M. Ribeiro, Characterization of the variable exponent Sobolev norm without derivatives, Commun. Contemp. Math. 19 (2017), 1650022, 13 pp.

[26] V. Kokilashvili and M. Krbec, Weighted Inequalities in Lorentz and Orlicz Spaces, World Scientific Publishing Co., Inc., River Edge, NJ, 1991.

[27] M. A. Krasnosel'skĭ and Ja. B. Rutickii, Convex Functions and Orlicz Spaces, Translated from the first Russian edition by Leo F. Boron, P. Noordhoff Ltd., Groningen 1961.

[28] J. Musielak, Orlicz Spaces and Modular Spaces, Lecture Notes in Mathematics, 1034, Springer-Verlag, Berlin, 1983.

[29] H. Nakano, Topology of Linear Topological Spaces, Maruzen Co., Ltd., Tokyo, 1951.

[30] H. Nakano, Modulared Semi-Ordered Linear Spaces, Maruzen Co., Ltd., Tokyo, 1950.

[31] T. Ohno and T. Shimomura, Musielak-Orlicz-Sobolev spaces on metric measure spaces, Czechoslovak Math. J. 65(140) (2015), 435-474.

[32] T. Ohno and T. Shimomura, Musielak-Orlicz-Sobolev spaces with zero boundary values on metric measure spaces, Czechoslovak Math. J. 66(141) (2016), 371-394. 
[33] W. Orlicz, Über eine gewisse Klasse von Räumen vom Typus B, Bull. Int. Acad. Pol. Ser. A 8 (1932), 207-220.

[34] M. M. Rao and Z. Ren, Applications of Orlicz Spaces, Marcel Dekker, New York, 2002.

[35] M. M. Rao and Z. Ren, Theory of Orlicz Spaces, Marcel Dekker, New York, 1991.

[36] M. Squassina and B. Volzone, Bourgain-Brézis-Mironescu formula for magnetic operators, C. R. Math. Acad. Sci. Paris 354 (2016), 825-831.

[37] B. O. Turesson, Nonlinear Potential Theory and Weighted Sobolev Spaces, Lecture Notes in Mathematics, 1736, Springer-Verlag, Berlin, 2000.

[38] D. Yang, Y. Liang and L. D. Ky, Real-variable Theory of Musielak-Orlicz Hardy Spaces, Lecture Notes in Mathematics, 2182, Springer, Cham, 2017.

[39] A. Youssfi and Y. Ahmida, Some approximation results in Musielak-Orlicz spaces, arXiv: 1708.02453.

Sibei Yang

School of Mathematics and Statistics, Gansu Key Laboratory of Applied Mathematics and Complex Systems, Lanzhou University, Lanzhou 730000, People's Republic of China

E-mail: yangsb@lzu.edu.cn

Dachun Yang (Corresponding author) and Wen Yuan

Laboratory of Mathematics and Complex Systems (Ministry of Education of China), School of Mathematical Sciences, Beijing Normal University, Beijing 100875, People's Republic of China

E-mails: dcyang@bnu.edu.cn (D. Yang)

wenyuan@bnu. edu.cn (W. Yuan) 